\title{
Estudio prospectivo sobre la utilidad de la ecografía de control tras la realización de pruebas invasivas hepáticas: biopsia hepática y punción aspiración con aguja fina (PAAF)
}

\author{
E. Carrera Alonso, M. García González, P. Valer López-Fando, G. Plaza Palacios, A. López San Román, \\ L. Gil Grande y J. M. Milicua Salamero
}

Departamento de Gastroenterología. Hospital Ramón y Cajal. Madrid

\section{RESUMEN}

Objetivo: establecer si es necesario realizar una ecografía de control a todos los pacientes sometidos a una biopsia hepática o una punción aspiración con aguja fina, para detectar posibles complicaciones con o sin repercusión clínica.

Material y métodos: tras la realización de una biopsia hepática o una punción aspiración con aguja fina según el protocolo habitual, se mantiene al paciente en observación durante 24 horas, realizándose en ese momento una ecografía a todos los pacientes aunque no presenten datos clínicos de complicación.

Resultados: se llevaron a cabo 298 biopsias hepáticas y 98 punciones mediante aguja fina. Presentaron complicaciones un total de 37 pacientes (9,34\%), de las cuales 36 (9,09\%) fueron complicaciones menores en forma de dolor, síncope vasovagal o hemorragia leve y $1(0,25 \%)$ complicación mayor en forma de hemorragia grave. De las 396 exploraciones tan sólo uno de los casos presentó una complicación detectada en la ecografía (hematoma intraparenquimatoso) encontrándose asintomático.

Conclusiones: la baja incidencia de complicaciones, que cursan de forma asintomática, y la buena evolución de las mismas hacen poco rentable la realización de ecografía de control tras la realización de dichas técnicas diagnósticas, siendo necesaria tan sólo en el caso de sospecha clínica de complicación.

Palabras clave: Biopsia hepática. Punción aspiración aguja fina. Complicaciones. Ecografía.

\begin{abstract}
Objective: to determine the need to perform ultrasound scans to all patients after liver biopsy or fine-needle aspiration (FNA) in order to detect complications with or without symptoms.

Material and methods: after liver biopsy or FNA using a regular protocol the patient is observed for 24 hours at the hospital, and all patients undergo an abdominal sonography at that time even in the absence of evident complications.

Results: 298 liver biopsies and 98 FNAs were performed. There were complications in 37 patients (9.34\%): 36 (9.09\%) were minor complications such as pain, vasovagal episodes, or small bleeding, and $1(0.25 \%)$ was a major complication with severe hemorrhage. Only 1 out of all 396 procedures had a complication detected by ultrasounds (intrahepatic hematoma) while the patient was asymptomatic.

Conclusions: the low incidence of complications occurring without symptoms, and their favorable course suggest that routine ultrasonography is not necessary after these techniques, and that it should be only performed when a complication is suspected.
\end{abstract}

Key words: Liver biopsy. Fine-needle biopsy. Complications. Ultrasound.

Carrera Alonso E, García González M, Valer López-Fando P, Plaza Palacios G, López San Román A, Gil Grande L, Milicua Salamero JM. Estudio prospectivo sobre la utilidad de la ecografía de control tras la realización de pruebas invasivas hepáticas: biopsia hepática y punción aspiración con aguja fina (PAAF). Rev Esp Enferm Dig 2007; 99: 128-131.

Recibido: 21-04-06.

Aceptado: 05-12-06.

Correspondencia: Elisa Carrera Alonso. Departamento de Gastroenterología. Hospital Ramón y Cajal. Ctra. de Colmenar, km 9,100. 28034 Madrid. e-mail: elisacar@terra.es.

\section{INTRODUCCIÓN}

La biopsia hepática $(\mathrm{BH})$ y la punción aspiración con aguja fina (PAAF) son dos técnicas fundamentales realizadas de forma rutinaria en el estudio de las enfermedades hepáticas, con una alta rentabilidad diagnóstica y una buena tolerancia por los pacientes. Sin embargo a pesar de hacerse de forma correcta y respetando sus contraindi- 
caciones no están exentas de complicaciones. Por este motivo las indicaciones de dicha técnica están siendo modificadas y sustituidas por otras exploraciones alternativas. En el caso de la hepatitis por el virus $\mathrm{C}$, el examen histológico ayuda a valorar aquellos candidatos al tratamiento antiviral. Sin embargo se están desarrollando pruebas serológicas y otros procedimientos no invasivos que puedan correlacionarse con el grado de fibrosis y así sustituir la biopsia hepática. En el caso del virus de la hepatitis B, la biopsia puede ser de menor utilidad para determinar los candidatos al tratamiento, aportando importante información los estudios analíticos y serológicos. Es controvertida la necesidad de la biopsia para el estudio de la esteatosis hepática ya que se puede realizar el diagnóstico de presunción mediante otras exploraciones no invasivas y no existe un tratamiento efectivo (1).

A pesar de lo dicho anteriormente, en el momento actual, tanto la biopsia como la PAAF son dos herramientas básicas en el estudio de las enfermedades hepáticas y resultan insustituibles a pesar de los avances técnicos. En torno al 5,9\% de los pacientes sometidos a estos procedimientos pueden sufrir complicaciones, generalmente menores (2). Dentro de las complicaciones las de mayor importancia son las de tipo hemorrágico (tras $\mathrm{BH}$ 0,35-0,5\%, tras PAAF 0,13-2,5\%) (3-6). Diferentes estudios han manifestado que el $61 \%$ de las complicaciones ocurren en las primeras dos horas de la realización de la exploración y el $96 \%$ en las primeras 24 horas (7).

El objetivo de nuestro estudio es establecer si es necesario realizar una ecografía de control a todos los pacientes sometidos a una BH o una PAAF, para detectar posibles complicaciones con o sin repercusión clínica.

\section{MATERIAL Y MÉTODOS}

Se realizó un estudio prospectivo que incluyó a 396 pacientes que fueron sometidos a una biopsia hepática o PAAF con obtención de muestra representativa para estudio en nuestra Unidad de Ecografía del Servicio de Gastroenterología a lo largo de un año natural. De todos ellos, 298 fueron BH y 98 PAAF. Todos los pacientes firmaron el consentimiento informado antes del procedimiento. Se contraindicó la exploración con una cifra de plaquetas inferior a $50.000 / \mathrm{mm}^{3}$ o una actividad de protrombina menor de $50 \%$.

Todos los procedimientos se realizaron según el protocolo habitual que consiste en ayuno de más de 6 horas, e inmediatamente antes del procedimiento la administración de 0,5-1 $\mathrm{ml}$ de atropina subcutánea. El punto óptimo de punción se localizó bajo control ecográfico. En el caso de la biopsia la localización del punto se siguió del marcado del mismo y a continuación la punción no guiada. Para la PAAF se empleó control ecográfico directo. Posteriormente se instiló el anestésico local (mepivacaína $1 \%$ ). Se utilizaron agujas de Tru-cut ${ }^{\boxplus}$ manuales de $14 \mathrm{G}$ (Baxter, Valencia España) o de Menghini ${ }^{\circledast}$ (Hepafix $^{\circledast}$
Luer lock, Braun,) para la BH y agujas de 25G (BD Spinal Needle 25 GA Becton Dickinson) para la PAAF. Los pacientes permanecieron en observación hospitalaria durante un periodo de 18 horas. Tras la exploración se tomaron las constantes cada 15 minutos durante las primeras dos horas, cada 30 minutos en las siguientes dos horas y posteriormente cada hora durante las siguientes 24 horas.

Se realizó una ecografía de control a todos los pacientes a las 24 horas de la exploración si se mantenían asintomáticos, haciéndose de forma urgente en caso de sospecha de complicación (TA sistólica menor de $100 \mathrm{mmHg}$, dolor intenso, mareo, náuseas o vómitos).

Las complicaciones se clasificaron en dos grupos:

1. Complicaciones menores:

-Dolor en la zona de punción o irradiado al hombro derecho que precisó analgesia sin objetivarse complicaciones en la ecografía.

- Cuadro de hipotensión con ecografía normal, considerado como síncope vasovagal.

-Hemorragia que no cumple los criterios de complicación mayor.

2. Complicaciones mayores:

- Hemorragia en forma de hematoma intrahepático, subcapsular o hemoperitoneo que requiere transfusión o una intervención quirúrgica.

-Punción de otros órganos.

Tabla I. Resultados de las complicaciones registradas

\begin{tabular}{llccc}
\hline \multicolumn{2}{c}{ Complicaciones } & BH & PAAF & Total \\
\hline $\begin{array}{l}\text { Complicaciones } \\
\text { menores }\end{array}$ & Dolor & 25 & 1 & 26 \\
& Síncope vasovagal & 2 & 0 & 2 \\
& Hemorragia leve & $\begin{array}{c}6 \text { con dolor } \\
1 \text { asintomática }\end{array}$ & 1 & 8 \\
\hline $\begin{array}{l}\text { Complicaciones } \\
\text { mayores }\end{array}$ & Hemorragia grave & 1 & 0 & 1 \\
\hline Total & 35 & 2 & 37 \\
\hline
\end{tabular}

BH: biopsia hepática; PAAF: punción aspiración con aguja fina.

\section{RESULTADOS}

Presentaron complicaciones un total de 37 pacientes $(9,34 \%)$.

-Complicaciones menores:

Dos pacientes $(0,50 \%)$ presentaron un cuadro de hipotensión tras la realización de la $\mathrm{BH}$, no objetivándose en la ecografía complicaciones ni anemización, por lo que se consideró como un síncope vasovagal.

De todos los pacientes sometidos a BH $25(8,38 \%)$ precisaron analgesia para el control del dolor, mientras que esto ocurrió sólo en uno $(1,02 \%)$ de los pacientes sometidos a PAAF. En ninguno de ellos pudo la ecografía objetivar complicaciones secundarias a la exploración. 
Un total de 34 pacientes $(8,58 \%)$ presentaron dolor tras la exploración de los cuales en $8(2,02 \%)$ se observó un hematoma en la ecografía, mientras que los 26 (25 BH y 1 PAAF) restantes tenían una ecografía sin alteraciones $(6,56 \%)$.

Se observaron $8(2,02 \%)$ hemorragias leves en forma de hematoma intraparenquimatoso, 7 tras la realización de BH $(2,34 \%)$ y una tras PAAF $(1,02 \%)$. De estos uno de ellos cursó sin ninguna sintomatología y el resto con dolor abdominal sin shock ni hipotensión. Todos ellos se resolvieron con tratamiento conservador sin precisar transfusión sanguínea. El paciente que cursó sin ninguna sintomatología tenía un hematoma de $12 \mathrm{~mm}$ de tamaño. Los otros 7 pacientes presentaron hematomas de entre 8 y $20 \mathrm{~mm}$ de tamaño. No se observaron diferencias importantes entre los tamaños de los hematomas de los pacientes que presentaron síntomas y los que cursaron asintomáticos.

\section{-Complicaciones mayores:}

Se registró una $(0,25 \%)$ complicación mayor que presentó dolor abdominal asociado a hipotensión tres horas después de la realización de la biopsia. En la ecografía se observó un hemoperitoneo que precisó transfusión de dos concentrados de hematíes, pero se resolvió espontáneamente sin necesidad de tratamiento quirúrgico.

Del total de las ecografías realizadas de forma rutinaria sin ninguna sintomatología asociada que sugiriese una complicación, tan sólo una $(0,27 \%)$ puso de manifiesto un hematoma intrahepático que se resolvió de forma conservadora. En las 359 ecografías restantes $(99,7 \%)$ no se detectó ninguna alteración secundaria al procedimiento.

\section{DISCUSIÓN}

Es difícil determinar cuál es la tasa global de complicaciones tras la realización de la $\mathrm{BH}$ o PAAF ya que la mayoría de los estudios son retrospectivos y en muchos de ellos no quedan recogidos algunos síntomas como por ejemplo el dolor.

Además no existe de forma consensuada una clasificación de las complicaciones entre menores y mayores y no queda establecido si la existencia de un hematoma intrahepático que curse de forma asintomática debe ser incluido en una de estas dos formas.

En nuestra serie la tasa global de complicaciones (definidas previamente) tras la realización de una $\mathrm{BH}$ fue del $11,7 \%$, siendo las más frecuentes las complicaciones menores en forma de dolor en la zona de punción, cuadros vagales o hemorragias leves.

El dolor es sin duda la complicación más frecuente tras la realización de una $\mathrm{BH}$, que según algunos estudios aparece hasta en el 30\% de los pacientes, siendo este moderado o severo en el 3 y $1,5 \%$ respectivamente (8). En nuestra serie el dolor en la zona de punción sin alteraciones ecográficas, fue también la complicación más frecuente, precisando analgesia hasta el 8,9\% de los pacientes.
La PAAF realizada para la obtención de material histológico o citológico se ha extendido para el diagnóstico de lesiones focales hepáticas, especialmente en el diagnóstico del hepatocarcinoma. Se trata de una técnica muy sensible y específica, pero no está exenta de efectos adversos. La tasa de hemorragia descrita tras la realización de una PAAF se encuentra entre el 0,13-2,5\% según las series $(5,6)$.

Otra de las complicaciones importantes en el caso de la PAAF de lesiones malignas es el riesgo que tiene de diseminación en el trayecto de la punción realizada. Aunque la incidencia real no se conoce, el riesgo de diseminación tumoral es de 0,006\% aproximadamente (9). En nuestro caso dicha complicación no se ha tenido en cuenta ya que la diseminación tumoral se detecta generalmente de forma clínica a los meses o incluso años de la realización de la técnica y este no era el objetivo de nuestro estudio (10).

En nuestra serie la tasa global de complicaciones tras la PAAF fue del 2,04\%, todas ellas clasificadas en el grupo de complicaciones menores (una hematoma intraparequimatoso y un paciente precisó analgesia sin alteraciones ecográficas).

Dentro de las complicaciones mayores la hemorragia es la más importante. La tasa de hemorragia es muy variable según las series y dependiendo esta de algunos factores como por ejemplo la existencia de una cirrosis avanzada, hepatitis aguda fulminante, el tratamiento con corticosteroides, infecciones asociadas, las alteraciones de la coagulación, la administración de heparina el día previo a la biopsia o la insuficiencia renal crónica $(11,12)$. No existen estudios suficientemente evaluados que demuestren la disminución de las complicaciones en función de la postura tras la biopsia, la analgesia utilizada o del uso de sellado mediante sustancias como pegamento de fibrina $\left(\right.$ Tissucol $\left.^{\circledR}\right)(13,14)$. Según la literatura se producen hemorragias significativas hasta en el $0,5 \%$ de los casos (4). Sin embargo es mucho más frecuente la presencia de pequeños hematomas a nivel intrahepático o subcapsular, que se detectan al realizar una ecografía de control a las 24 horas del procedimiento invasivo.

En este sentido es importante determinar cuál es el significado clínico real de estos hematomas y qué porcentaje de los pacientes que lo desarrollan van a presentar síntomas que hagan sospechar dicha complicación. Estos datos son importantes, para establecer si es necesaria la realización de una ecografía de control a todos los pacientes a los que se les practica un procedimiento invasivo hepático, lo que supondría un elevado coste sanitario y una sobrecarga para las unidades de ecografías.

Según algunos estudios realizados previamente, hasta el $20 \%$ de los pacientes sometidos a una BH presentan hematomas intraparenquimatosos o subcapsulares. De estos el 16,7\% cursaron de forma asintomática, presentando tan sólo el 1,7\% síntomas que sugerían la existencia de una complicación (15). En otros estudios la tasa de esta complicación es mucho menor, detectándose me- 
diante gammagrafía hepática en el 7\% (16) y tras TC sin contraste en el 3,3\% (17).

En nuestra serie el $2,27 \%$ de los procedimientos $(\mathrm{BH}+$ PAAF) presentaron una complicación hemorrágica, de las cuales 8 fueron en forma de hematoma intraparenquimatoso. De las 396 exploraciones realizadas, tan sólo uno de los casos presentó una complicación ecográfica encontrándose el paciente asintomático. Dicha complicación fue en forma de hematoma intrahepático con un tamaño de $12 \mathrm{~mm}$.

La baja frecuencia de complicaciones graves asintomáticas y la buena evolución de las complicaciones hemorrágicas sintomáticas hacen que sea poco rentable la realización de una ecografía rutinaria a las 24 horas de una biopsia hepática o PAAF, siendo sólo necesaria en caso de sospecha de complicación (hipotensión, dolor o anemización).

\section{BIBLIOGRAFÍA}

1. Campbell MS, Reddy KR. Review article: the evolving role of liver biopsy. Aliment Pharmacol Ther 2004; 20: 249-59.

2. Perrault J, McGill DB, Ott BJ, Taylor WF. Liver biopsy: complications in 1000 inpatients and outpatients. Gastroenterology 1978; 74: 103-6.

3. McGill DB, Rakela J, Zinsmeister AR, Ott BJ. A 21 years of experience with major haemorrhage after percutaneous liver biopsy. Gastroenterology 1990; 99: 1396-400.

4. Knauer MC. Percutaneous biopsy of the liver as a procedure for out- patients. Gastroenterology 1978; 74: 101-2.

5. Bret PM, Labadie M, Bretabnolle M, Paliard D, Fond A, Valette PJ, et al. Hepatocellular carcinoma: diagnosis by percutaneous fine needle biopsy. Gastrointest Radiol 1988; 13: 253-5.

6. Gazelle S, Haaga JR, Rowland DY. Effect of needle gauge, level of anticoagulation, and target organ on bleeding associated with aspiration biopsy. Radiology 1992; 183: 509-13.

7. Bravo A, Sheth S, Chopra S. liver biopsy. N Engl J Med 2001; 344: 495-500.

8. Grant A, Neuberger J. Guidelines on the use of liver biopsy in clinical practice. Gut 1999; 45 (Supl. IV): IV1-IV11.

9. Smith EH. Complications of percutaneous abdominal fine needle biopsy. Radiology 1991; 178: 253-8.

10. Ebara M. Complications of fine needle biopsy for focal liver lesions. Hepatol Res 2005; 3: 3-4.

11. Terjung B, Lemnitzer I, Dumoulin F, et al. Bleeding complications after percutaneous liver biopsy. Digestion 2003; 67: 138-45.

12. Ozdogan M, Ozgur O, Boyactoglu S, Coskun M, Kart H, Ozdal S, et al. Percutaneous liver biopsy. Complications in patients with chronic renal failure. Nephron 1996; 74: 442-3.

13. Hyun C, Beutel V. Prospective randomized trial of post-liver biopsy recovery positions. J Clin Gastroenterol 2005; 39: 328-32.

14. Albeniz Arbizu E, López San Román A, García González M, Foruny Olcina JR, García-Hoz Rosales F, Barcena Marugan R, et al. Fibrin-glue sealed liver biopsy in patients with a liver transplantation or in liver transplantation waiting list: preliminary results. Transplant Proc 2003; 35: 1911-2.

15. Sugano S, Sumino Y, Hatori T, Mizugami H, Kawafune T, Abei T. Incidence of ultrasound-detected intrahepatic hematomas due to Tru-cut needle liver biopsy. Dig Dis Sci 1991; 36: 1229-33.

16. Raines DR, Van Heertum RL, Johnson LF. Intrahepatic hematoma: A complication of percutaneous liver biopsy. A report on the incidence of postbiopsy scan defects. Gastroenterology 1974; 67: 284-9.

17. Malone DE, McCormick PA, O’Donoghue DP, MacErlean DP. Computed tomography of the liver alter biopsy. Clin Radiol 1978; 38: 97-9. 